\title{
Exploring Eye-Gaze Wheelchair Control
}

\section{Araujo, Jacopo M.; Zhang, Guangtao; Hansen, John Paulin Paulin; Puthusserypady, Sadasivan}

\section{Published in:}

Proceedings of ACM Symposium on Eye Tracking Research and Applications

Link to article, DOI:

$10.1145 / 3379157.3388933$

Publication date:

2020

Document Version

Publisher's PDF, also known as Version of record

Link back to DTU Orbit

Citation $(A P A)$ :

Araujo, J. M., Żhang, G., Hansen, J. P. P., \& Puthusserypady, S. (2020). Exploring Eye-Gaze Wheelchair Control. In Proceedings of ACM Symposium on Eye Tracking Research and Applications (pp. 1-8). [16] Association for Computing Machinery. Acm Symposium on Eye Tracking Research and Applications https://doi.org/10.1145/3379157.3388933

\section{General rights}

Copyright and moral rights for the publications made accessible in the public portal are retained by the authors and/or other copyright owners and it is a condition of accessing publications that users recognise and abide by the legal requirements associated with these rights.

- Users may download and print one copy of any publication from the public portal for the purpose of private study or research.

- You may not further distribute the material or use it for any profit-making activity or commercial gain

- You may freely distribute the URL identifying the publication in the public portal 


\section{Exploring Eye-Gaze Wheelchair Control}

\author{
Jacopo de Araujo \\ Technical University of Denmark \\ Kgs. Lyngby, Denmark \\ jacopomattiadearaujo@gmail.com \\ Guangtao Zhang \\ Technical University of Denmark \\ Kgs. Lyngby, Denmark \\ guazha@dtu.dk
}

\author{
John Paulin Hansen \\ Technical University of Denmark \\ Kgs. Lyngby, Denmark \\ jpha@dtu.dk \\ Sadasivan Puthusserypady \\ Technical University of Denmark \\ Kgs. Lyngby, Denmark \\ sapu@dtu.dk
}

\begin{abstract}
Eye-gaze may potentially be used for steering wheelchairs or robots and thereby support independence in choosing where to move. This paper investigates the feasibility of gaze-controlled interfaces. We present an experiment with wheelchair control in a simulated, virtual reality (VR) driving experiment and a field study with five people using wheelchairs. In the VR experiment, three control interfaces were tested by 18 able-bodied subjects: (i) dwell buttons for direction commands on an overlay display, (ii) steering by continuous gaze point assessment on the ground plane in front of the driver, and (iii) waypoint navigation to targets placed on the ground plane. Results indicate that the waypoint method had superior performance, and it was also most preferred by the users, closely followed by the continuous-control interface. However, the field study revealed that our wheelchair users felt uncomfortable and excluded when they had to look down at the floor to steer a vehicle. Hence, our VR testing had a simplified representation of the steering task and ignored an important part of the use-context. In the discussion, we suggest potential improvements of simulation-based design of wheelchair gaze control interfaces.
\end{abstract}

\section{CCS CONCEPTS}

- Human-centered computing $\rightarrow$ Usability testing; Interaction design; User interface design; Graphical user interfaces; Virtual reality; • Computing methodologies $\rightarrow$ Motion path planning.

\section{KEYWORDS}

gaze interaction, eye-tracking, gaze control, vehicle control, wheelchair, virtual reality, simulation, human-robot interaction, locked-in syndrome, amyotrophic lateral sclerosis, ALS/MND

Permission to make digital or hard copies of all or part of this work for personal or classroom use is granted without fee provided that copies are not made or distributed for profit or commercial advantage and that copies bear this notice and the full citation on the first page. Copyrights for components of this work owned by others than ACM must be honored. Abstracting with credit is permitted. To copy otherwise, or republish, to post on servers or to redistribute to lists, requires prior specific permission and/or a fee. Request permissions from permissions@acm.org.

ETRA '20 Adjunct, June 2-5, 2020, Stuttgart, Germany

(C) 2020 Association for Computing Machinery.

ACM ISBN 978-1-4503-7135-3/20/06 . \$ $\$ 15.00$

https://doi.org/10.1145/3379157.3388933
ACM Reference Format:

Jacopo de Araujo, John Paulin Hansen, Guangtao Zhang, and Sadasivan Puthusserypady. 2020. Exploring Eye-Gaze Wheelchair Control. In Symposium on Eye Tracking Research and Applications (ETRA '20 Adjunct), fune 2-5, 2020, Stuttgart, Germany. ACM, New York, NY, USA, 8 pages. https: //doi.org/10.1145/3379157.3388933

\section{INTRODUCTION}

People with severe damage to the central nervous system or motor neuron diseases such as amyotrophic lateral sclerosis (ALS) may lose motor control and in rare and extreme cases get a so-called locked-in syndrome (LIS)[Kiernan et al. 2011]. LIS patients are limited to functional use of their brain and eyes only. Gaze interaction and brain computer interfaces (BCI) have both been suggested as wheelchair-control methods for LIS patients and people with other complex motor challenges [Arai and Mardiyanto 2011; Barea et al. 2003; Bartolein et al. 2008; Eid et al. 2016; Elliott et al. 2019; Leeb et al. 2007; Lin et al. 2006; Matsumotot et al. 2001]. Gaze interaction is less expensive than $\mathrm{BCI}$, with data rates orders of magnitudes faster. They are also more operable and convenient to use for vehicle control than BCI is [et Al 2019; Sebastian Halder and Kansaku 2018; William W. Abbott 2011].

Gaze interactions are commonly used for communication by LIS-patients [Spataro et al. 2014], as they cannot use their voice and hands. The main challenge for safe and efficient gaze control interfaces is unintentional selection. This gives rise to the problem of distinguishing intended control actions from non-intended, a problem commonly referred to as the Midas touch dilemma [Hachem A. Lamti and Hugel 2019; Jacob 1991]. Advances in sensor technology and artificial intelligence (AI) may partly overcome this, for instance, by intelligent collision avoidance [Eid et al. 2016; Leaman and La 2017], but it is an open issue on how to design the user interface for semi-autonomous gaze-controlled vehicles.

Obstacle courses in simulated environments are frequently used for testing the usability of alternative wheelchair control. VR has also been applied for training and assessment of wheelchair control methods [Buxbaum et al. 2008; Harrison et al. 2000, 2002]. Design of gaze interfaces for navigating in VR has been studied extensively (e.g. [Stellmach and Dachselt 2012; Tanriverdi and Jacob 2000]). Gaze control of wheelchairs has been evaluated in VR [Ktena et al. 2015] as well as in real physical environments [Matsumotot et al. 2001; Wästlund et al. 2010]. Gaze-controlled robots has also been studied in VR (e.g. [Watson et al. 2016; Zhang and Hansen 2019]) 


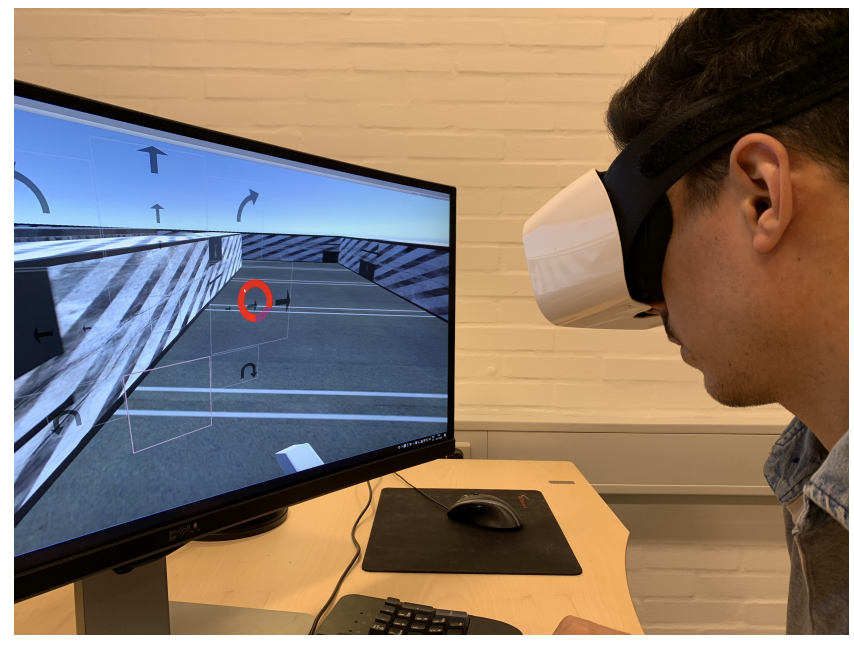

Figure 1: Experiment setup with the view from the headmounted gaze-tracking display shown on the monitor. The participant turns to the right by dwelling at an overlay key (indicated by a red progress circle)

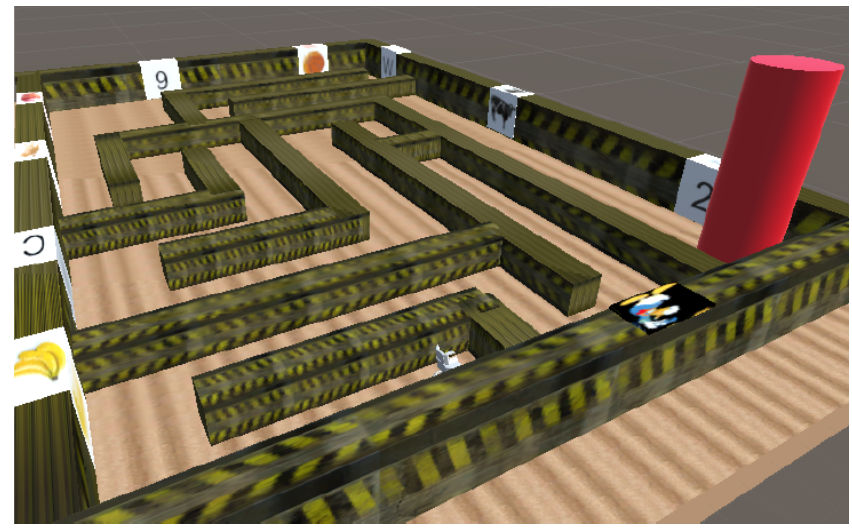

Figure 2: Example of an VR obstacle course from the experiment. Pictures on the walls are used for measures of situational awareness, and the red cylinder marks the end-goal

and in real environments (e.g. [Leaman and La 2017; Tall et al. 2009]).

Virtual simulation offers advantages compared to a full-scale physical test environment namely low cost, easy configurability, low risk of damage, high repeatability, and portability [Ktena et al. 2015; Talone et al. 2013]. However, it may also ignore important part of the driving situation. We therefore decided to investigate the feasibility of VR for the preliminary testing of gaze-controlled wheelchair interfaces. Since VR is a model that may simplify parts of the steering task and ignore some of the use-context compared to a real-life situation, we conducted a follow-up field study with five people with motor disabilities, i.e. our target group. The main purpose was to improve the fidelity of simulation-based development of gaze-control of wheelchairs and robots.

\section{VR WHEELCHAIR GAZE INTERFACE}

The wheelchair simulation developed for our study places the subject in a virtual wheelchair while equipped with a head-mounted display (HMD) that has gaze tracking built into it. Figure 1 shows the experimental setup with a user wearing the headset as well as the VR world shown on the monitor in front of him. Figure 2 shows a birds-eye view of the obstacle courses. The images on the walls were used to measure situational awareness. The red cylinder marks the goal. The Unity 3D code for our experiment are available online $^{1}$.

Three different gaze-controlled interfaces were tested in our study.

\subsection{Overlay dwell-button interface}

The overlay interface has nine buttons yielding 16 commands with a $500 \mathrm{~ms}$ gaze dwell activation. Feedback on the dwell time activation is provided by a circular progress icon. Buttons are used for selecting combinations of motor torques and steering angles that are represented by arrow figures on the buttons. The forward button has three intensities of motor torque, and the left/right buttons has three levels of steering angles. Once a control action is executed, it will continue on this torque until there is a reset on the center-button (equivalent to a neutral-gear), or until it gets overwritten by another command. The brake button is a big button in the lower hemisphere and is marked with a red contour. When the vehicle stands still, the brake button will make the vehicle go in reverse. Visual inspection of the environment can only be done outside the overlay or with short fixations below the dwell-time threshold (see Figure 3).

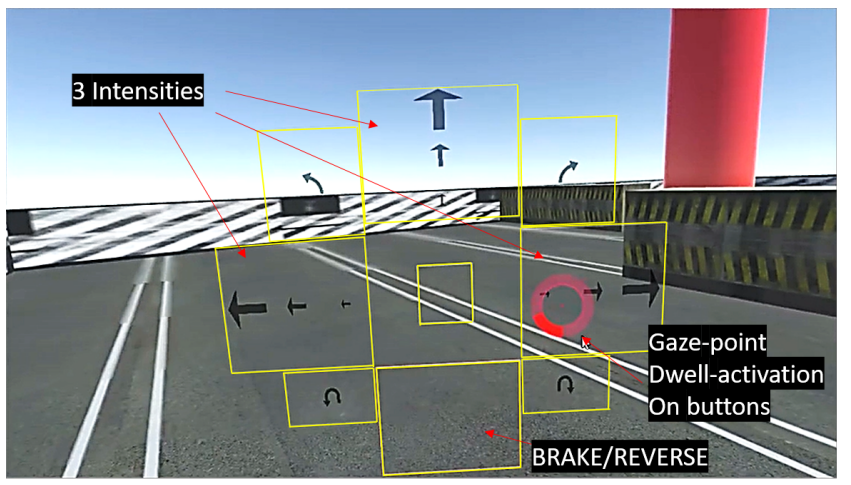

Figure 3: Overlay control interface with referring red arrows and explaining text boxes. (For illustrative purposes, the original transparent gray borders of the GUI have been highlighted in yellow)

\subsection{Continuous-control interface}

With this , the steering is done by measuring the gaze point intersection with the ground plane in relation to the driver's current position. This vector's depth and horizontal values are mapped through a linear and exponential function to output motor torque

\footnotetext{
${ }^{1}$ https://github.com/wheelchairdrivingsimulation/WheelchairDrivingSimulation
} 
and a steering angle, respectively. The mapping functions were defined by upper and lower thresholds as well as slope, which were then fine-tuned through a pilot study with three subjects. Furthermore, a graphic user interface (GUI) area for braking and reversing (with rotation) is provided in the upper hemisphere; the brake button makes the wheelchair reverse providing it's already standing still. Below the brake and reverse buttons, there is an orientation space that resets any control action. This space can be used for orientation purposes and to stop the continuous control. All four areas are marked with a box and transparent text (see Figure 4).

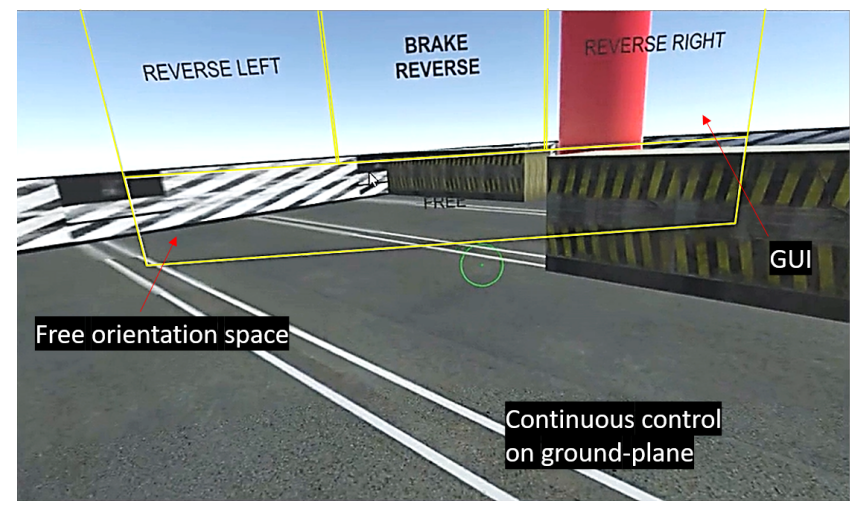

Figure 4: Continuous-control interface with referring red arrows and text boxes. (For illustrative purposes, the original transparent gray borders of the GUI have been highlighted in yellow)

\subsection{Semi-autonomous waypoint interface}

By utilizing a navigation and path finding module included in the VR software to direct the motion of the wheelchair along the fastest route to a waypoint, the interface is semi-autonomous. Waypoints are set on the ground plane by a $750 \mathrm{~ms}$ dwell-activation with a progress circle for feedback. This interface has an overlay GUI in the upper hemisphere for rotation and braking. As in the previous case, the brake button reverses if the wheelchair is standing still. Orientation can be done by avoiding looking on the interactive ground-plan or by gazing for less than the dwell-time (see Figure $5)$.

\section{METHODS}

\subsection{Participants}

The experiments were conducted with 18 subjects of which 13 were male. The mean age was 31 years \pm 11.5 . The participants were recruited from our university and from our personal network The subjects had little or no experience with VR, and none had experience with gaze interaction. Two subjects did not complete all the control interface conditions, and their data were excluded from the analysis. Four of the subjects used their own glasses inside the HMD, and no issues with this were reported.

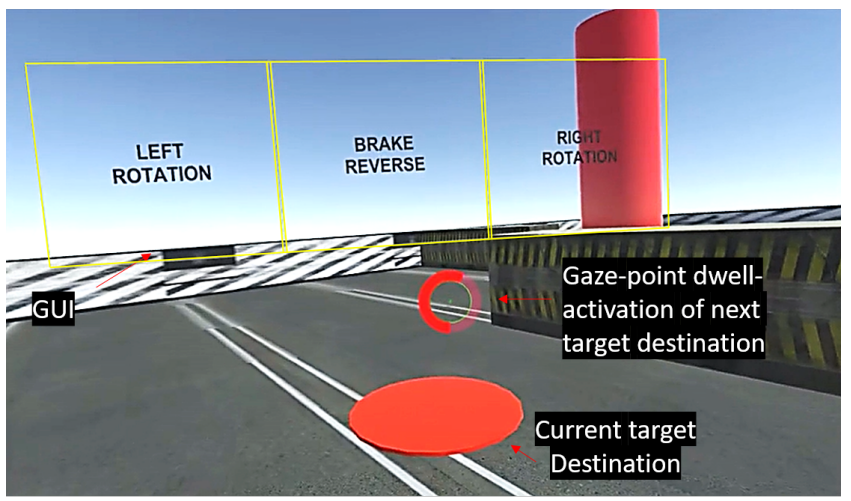

Figure 5: waypoint control interface with referring red arrows and text boxes. (For illustrative purposes, the original transparent gray borders of the GUI have been highlighted in yellow)

\subsection{Apparatus}

A FOVE virtual reality head-mounted display was used for the experiments. The HMD provides inertial measurement unit (IMU) orientation tracking of field of view (FOV) along with IR-based binocular gaze tracking. The eye-tracking sampling rate is 120 $\mathrm{Hz}$ fps and the manufacturers indicate a tracking accuracy to be $<1$ degree of the visual angle. The WQHD OLED display has a resolution of $2560 \times 1440$ with a frame rate of $70 \mathrm{fps}$. The spans up to 100 degrees horizontally. The environment was developed in Unity (ver. 0.16.0), a real-time development platform for 2D, 3D, VR, and AR. The simulation was run on a NVIDIA GTX 1080 grapic card.

\subsection{Procedure}

Upon arrival, participants were informed of the purpose and the procedure of the experiments and then asked to sign an informed consent form. They were then given an introduction to the control interfaces and the test courses, supported by a short video tutorial. The participants were informed of the main task of the test courses, specifically to reach the marked end-goal of the course as fast and with as few collisions as possible. Additionally, they were informed of a secondary task of test courses 4 and 6, being to remember the images appearing on the wall. This was done to measure their situational awareness while driving.

Before starting the experiments, the participants completed a questionnaire and were seated comfortably. The HMD was attached and gaze tracking was calibrated. The order of control interfaces (i.e. overlay, continuous, and waypoint) as well as the course conditions (difficulty levels 1-3) were determined by the experimental design (see Section 3.4). One training session was given for each interface condition prior to running the five test courses used for analysis. The participants were tasked with completing all difficulty levels with each interface condition before testing a new interface. Each course was marked completed when a participant parked at the end-goal (the large red cylinder). After completion of each of the sessions, the experimenter noted the number of images that the participant remembered correctly. In between the testing of each 
interface, the participants were encouraged to take a 10-minute break. After testing all interfaces, the participants completed a post-experimental questionnaire, ranking the interfaces in terms of intuitiveness, control, and reliability. They were also asked which interface they preferred. The full experiment took between one and a half to two hours per participant.

\subsection{Design}

The experiment used a $3 \times 3$ within-subject design with three control interfaces (overlay, continuous, and waypoint) and three difficulty levels (easy, medium, and hard). Each subject was to complete a training course with no obstacles followed by five test courses comprised of the three interfaces. A Latin square design was utilized for randomizing the order of the control interfaces among the participants, while the levels of difficulty were completed in a fixed order by each of the participants.

3.4.1 Easy conditions. : Courses 2 and 3 were the easy conditions. They had two fixed obstacles, 14 turns, and wide pathways. Both courses were expected to be completed three times to explore potential learning effects. This was only done for the easy condition, as the courses were relatively short (thus not pushing time constraints).

3.4.2 Medium condition. : Course 5 was the only course with a medium level of difficulty. It was designed with 10 turns and narrow pathways without barriers that would protect the wheelchair from falling off the road. This course incorporated a single obstacle that tested the driver's ability to brake as a reactive response to a roadblock spawning in front of the driver. The roadblock disappeared after 3 seconds, clearing the path toward the end-goal.

3.4.3 Hard conditions. : Courses 4 and 6 had the most difficult conditions. Like the medium-level course, they each had reactive braking episodes to respond to two obstacles. The courses had a total of 39 turns and were designed with narrow pathways. These courses had 10 images placed on the walls throughout the course, along with a secondary assignment to remember those. These courses were longer compared to the easy and medium ones. Five sligthly different versions of courses 4 and 6 were made with different images posted on the walls to measure situational awareness and/or with different positioning of the spawning roadblocks for testing reactive braking. The different course versions presented under the medium and hard conditions were determined by a Latin square design, changing the order for each participant and ensuring that no course version was encountered twice.

As explained above, the second and third courses were repeated three times, providing a total of 27 observations per participant (i.e., 3 repetitions of the 2 easy conditions +1 medium condition + 2 hard conditions $=9$, then $\mathrm{x} 3$ for each interface $=27$ ).

The dependent variables were task time (i.e., time to complete a trial), number of collisions, and situational awareness. Since collisions were not possible under the semi-autonomous waypoint interface, that utilized an anti-collision feature in the VR-software, they were only measured for the overlay and the continuous-control interface conditions.

\section{RESULTS}

\subsection{Task Time}

The grand mean for task time per trial was 155.3 seconds (s). For comparison, an expert user with standard game navigation on a keyboard had a grand mean of $40.5 \mathrm{~s}$. From fastest to slowest, the means were $82.2 \mathrm{~s}$ (waypoint), $136.6 \mathrm{~s}$ (continuous), and 246.9 (overlay). In terms of difficulty level, the easy condition $(M=74.81$, $S D=51.81)$ yielded lower mean task times compared to the medium condition $(M=179.68, S D=117.16)$ and the hard condition $(M=$ 223.51, $S D=150.94)$.

A repeated analysis of variance (ANOVA) showed that the main effect on task time was significant for interface $(F(2,150)=92.091$, $p<0.0001)$, and difficulty level $(F(4,150)=61.476, p<0.0001)$. Also, the interaction effect was significant for interface by difficulty level $(F(8,150)=7.0401, p<0.0001)$. (See figure Figure 6).

Post-hoc comparisons using Tukey's honest significant difference (HSD) test indicated that the interface waypoint $(M=82.2, S D$ $=47.97)$ was significantly faster than the continuous $(M=136.6, S D$ $=105.75)$, that was significantly faster than the overlay $(M=246.9$, $S D=159.26)$.

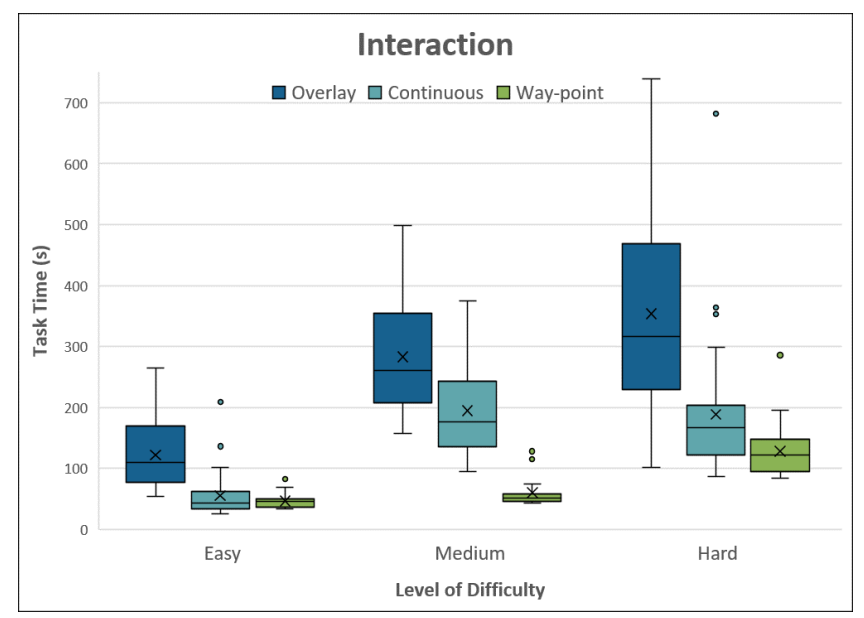

Figure 6: The interaction between interface condition and level of difficulty on task time.

\subsection{Collisions}

The waypoint interface was excluded from this analysis since the collision-avoidance feature automatically prevented all collisions. The grand mean was 2.5 collisions per trial. The main effect of the interfaces $(F(1,75)=26.1, p=0.0001)$ indicated that the continuous $(M$ $=1.6, S D=2.090)$ had significantly fewer collisions than the overlay $(M=3.3, S D=3.2)$. A repeated ANOVA test showed that the main effect of the difficulty level $(F(4,75)=20.619, p<0.0001)$ indicated significantly more collisions during the hard conditions, compared to the two other conditions. Post-hoc comparisons confirmed this was the case for all interfaces.(See Figure 7). 


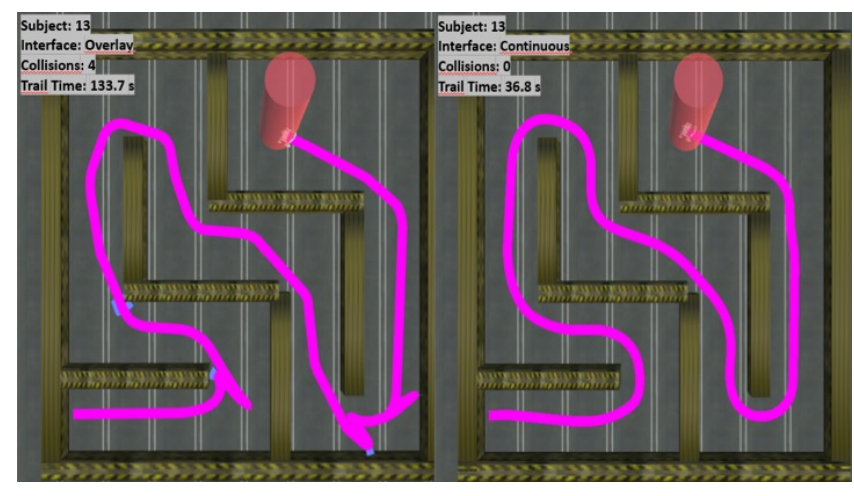

Figure 7: Track plots from subject 13. Left: Overlay display with four collisions and $133.7 \mathrm{~s}$ trail time . Right: Continuous-control interface with no collisions and $36.8 \mathrm{~s}$ trail time

\subsection{Situational Awareness}

Situational awareness was measured by the number of images a subject could recall upon completion of the level. There were a total of 10 images of recognizable objects placed throughout the obstacle course, and $75 \%$ of the subjects could remember up to 7 images regardless of the control interface. The grand mean was 5.3 images. A paired-samples $t$-test was conducted to compare the numbers of images remembered between the waypoint $(M=$ $6.2, S D=3.06)$, continuous $(M=5.23, S D=5.08)$ and overlay $(M=4.6, S D=5.01)$. There was no significant difference between the overlay and continuous control $(t(29)=-1.5006, p=0.1442)$, but there was a significant difference between the waypoint and both the overlay $(t(29)=-3.7882, p=0.0007)$ and the continuous $(t(29)=-2.1433, p=0.0406)$ control.

\subsection{User evaluations}

The user evaluation of the interfaces was conducted by a postexperimental questionnaire assessing the level of intuitiveness, control, and reliability of the interfaces on a Likert scale of 1-10. The grand mean was 6.56. From highest to lowest, the means were 7.63 (waypoint), 6.78 (continuous), and 5.28 (overlay). A repeated ANOVA test found significant differences among the interfaces $(F(2,96)=71.48, p<0.0001)$. In terms of intuitiveness, the waypoint $(M=7.47, S D=1.81)$ yielded significantly higher ratings than overlay $(M=5.88, S D=2.03)$, with no significant differences between the continuous $(M=6.77, S D=1.64)$ and any of the two other interfaces. In terms of control, the waypoint $(M=7.53, S D=1.38)$ and the continuous $(M=7.06, S D=1.14)$ both yielded significantly higher ratings than the overlay $(M=4.77, S D=2.31)$. In terms of reliability, the waypoint $(M=7.88, S D=1.36)$ yielded significantly higher ratings than the continuous $(M=6.53, S D=1.51)$ and overlay $(M=5.18, S D=1.94)$. There were no significant differences among the categories or interaction between categories and interfaces.

Subjects were finally asked to rank the three control interfaces against each other. From a total of 16 completed rankings, nine subjects ranked the waypoint interface first compared to six subjects

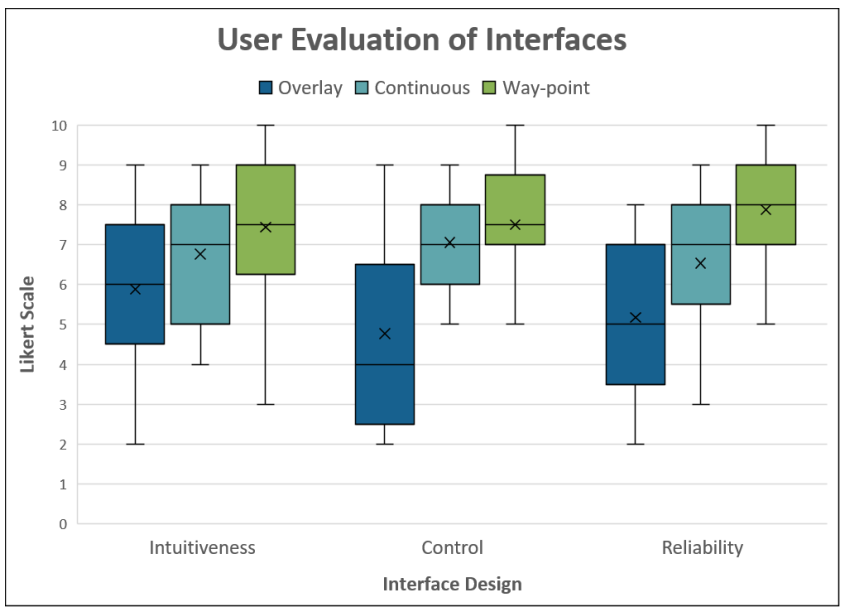

Figure 8: Subjective ratings of the interface design in terms of intuitiveness, control and reliability

who preferred the continuous-control while only one participant had a preference for the overlay-control.

\subsection{Summary of VR experiment}

The semi-autonomous waypoint control method with anti-collision outperformed the two other interfaces in terms of performance, situational awareness, and user preference.

The continuous-control interface performed similar to the waypoint interface under easy conditions, but fell behind under medium and hard conditions, implying shortcomings of the continuous interface's ability to perform cautious and accurate control. The overlay interface with GUI control performed the worst in test and on subjective ratings.

\section{FIELD OBSERVATIONS}

We also did a field study in a long-term care facility with 5 people, who use wheelchairs to compensate for motor disabilities caused by cerebral palsy or other neurological conditions. The observations were conducted as prototype experience sessions with different telerobots and control methods (hand, voice, and gaze). (See [Zhang and Hansen 2020] for further details). Here, we will report on only using gaze control of a telerobot by a continuous control interface and a waypoint interface, both much like those one used in the VR experiment. In the field study, the participants were controlling a telerobot, not a virtual wheelchair. The telerobot is also a wheeled device and the motion characteristics are much like those of the virtual wheelchair.

\subsection{Procedure}

The participants experienced gaze control in two different set-ups: Using the FOVE HMD with gaze pointing and steering with their gaze on a monitor by use of a Tobii $4 \mathrm{C}$ gaze tracker. One of the participants could only be calibrated for the monitor set-up not the FOVE. The monitor condition was done using a Wizard of $\mathrm{Oz}$ method [Salber and Coutaz 1993]. The experimenter stood behind 
the participant and moved a pointer to the locations of the user's gaze-point, which was shown on a monitor in front of them.

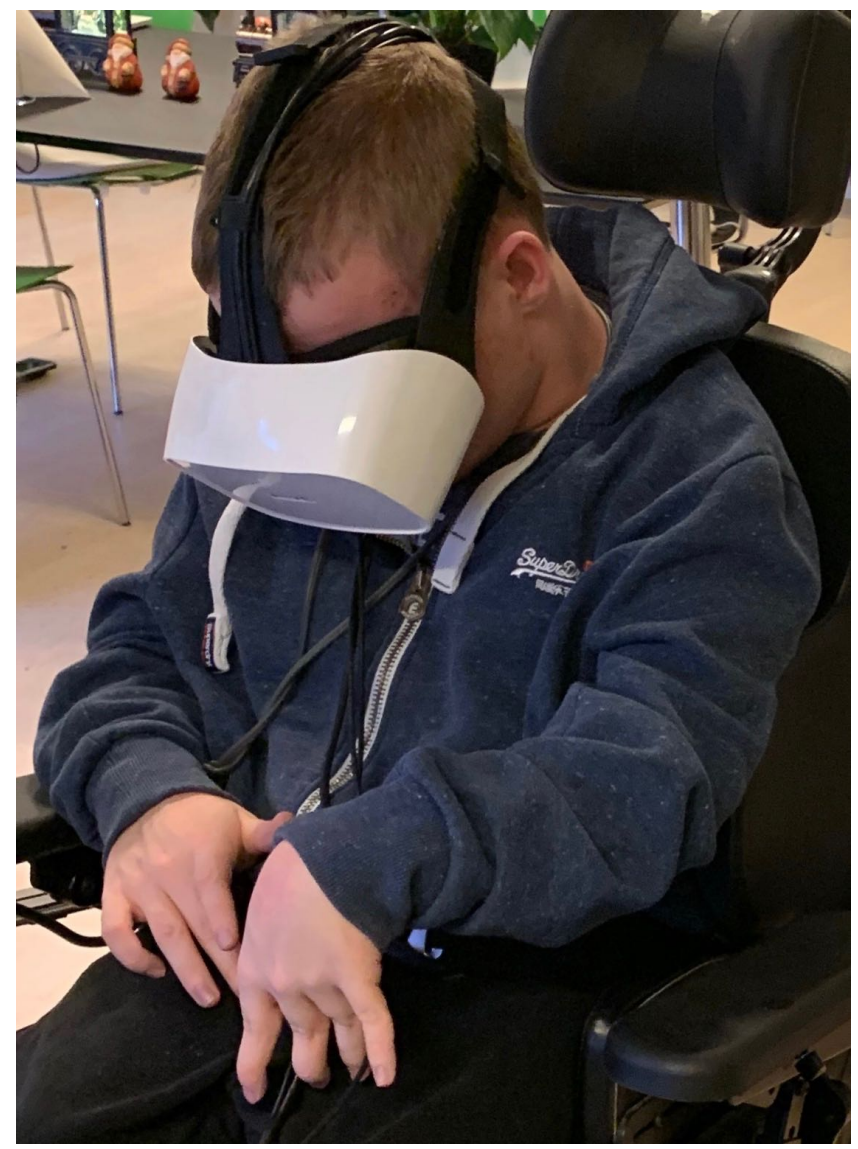

Figure 9: Testing gaze controlled navigation in a long-term care facility. Participants complained that looking to the floor-plan felt uncomfortable and made it difficult to see the surroundings.

The four participants who could use the FOVE were offered training sessions in a VR environment where they would then drive the robot around in a room about the size of the room they were in The participants were first trained to navigate a virtual robot in VR by conducting six trials. When using our VR simulator application for training, the simulated live video stream was generated from our 3D modeling in Unity. Once participants felt comfortable with the setup, they were asked to try driving a real robot. The two types of telerobots used were a modified PadBot with a 360-degree camera and a Double robot. Both of these would stream live video from the telerobot camera to the user's interface, on top of which the gaze navigation was done. Our application provided users with two ways of driving the telerobots, namely through continuous interface and through the waypoint control. In our case, waypoints could be marked with gaze dwelling at the floor for a set time.

The task was to drive around a table in the room, noticing what was on the table and greeting a person they would meet on their way All of the participants were able to do this using the continuous

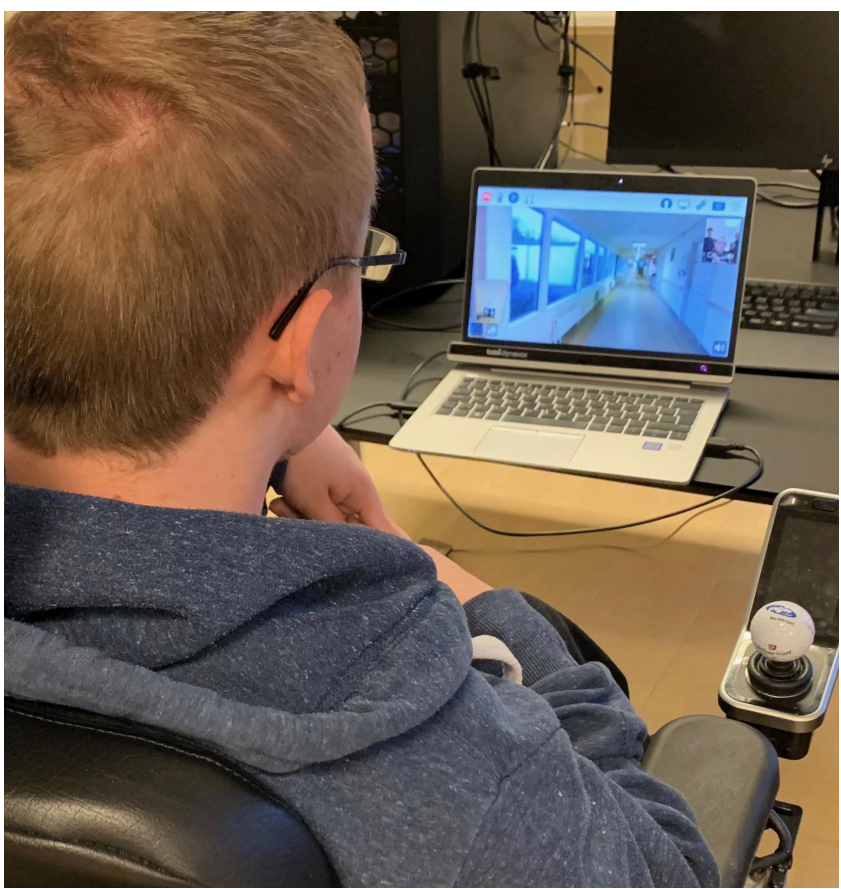

Figure 10: A participant driving a Double tele-robot in front of a screen with an tracker. The experimenter was standing behind the user and emulating his gaze movements by use of a joystick, applying a Wizard of $\mathrm{Oz}$ simulation of gaze interaction

interface but three of them had great difficulty doing the same task using the waypoint interface. In fact, only one person was able to drive all around the table using waypoint. This person was notably slower in driving with waypoints, and both he and the other subjects had several collisions with the table. We observed that the participants only moved with very short waypoint settings because they were afraid to loose control on a long leg. This also meant that they had to look far down on the ground, something that one of them remarked felt uncomfortable. Two other participants noticed that looking down made it difficult to observe the surroundings and engage in a social interaction (i.e., greeting the person they met). (See Figure 9).

\subsection{Observations}

When we asked the participants which of the gaze interaction methods they liked the most, the unanimous answer was the remote setup with a monitor (Figure 10). There were several reasons for this. First, it allowed them to attend to the people in the room while driving and to show their face uncovered at the telerobot monitor. Second, one participants found it limiting that he would not be able to put on the HMD himself or adjust it if needed. The remote setting, he imagined, would allow him to just drive up in front of the monitor and start using the telerobot without the need of assistance. Finally, some of the participants expected that the HMD would be uncomfortable to wear for an extended time. 


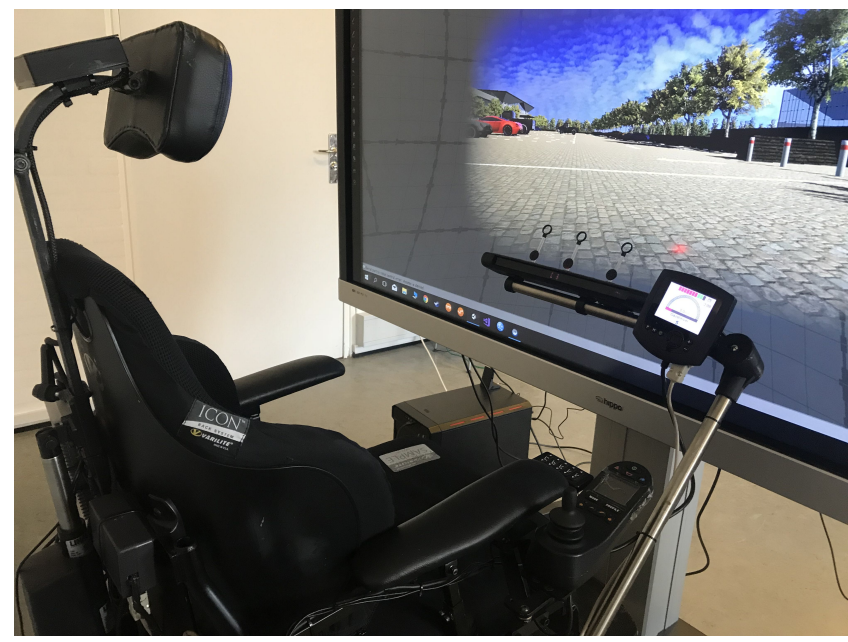

Figure 11: Testing the "GazeDriver" in a wheelchair simulator. The wheel-turns are transformed to movements in a VR game environment shown on the big monitor in front of the user. The user looks through the 3 circles above the tracker to steer left, straight,n or right.

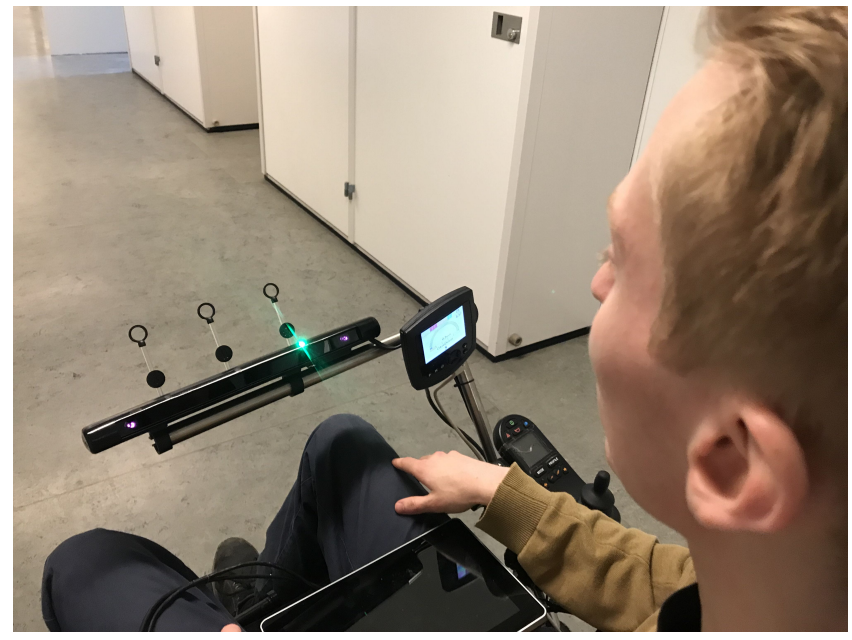

Figure 12: Driving with the "GazeDriver" in a real world felt much like driving it in the simulator. The green light indicate that the user is making a right turn because he looks through the right circle

\section{DISCUSSION}

Our experiment in the VR environment found superior performance and user preference for the semi-autonomous waypoint method. However, the wheelchair users in our field study did not like to be forced to look down on the floor to use this method. They only tried this method with a HMD set-up and they might have felt different, if the waypoint method had been used in a remote gaze tracking setup that would allow them to attend the surroundings. The training sessions applied a continuous-control interface. The participants may have felt more comfortable with the waypoint interface if they had been trained with this also. Without training, they tended to set the waypoints very close to each other, which forced them to look down a lot. The semi-autonomous functions of the waypoint interface that prevented the wheelchair from colliding was much appreciated by the participants in the VR experiment. However, collision avoidance in a complex and dynamic environment is an area open to research [Wang et al. 2013] and it is not likely that the "perfect" avoidance system in the VR environment will be available for real-world wheelchair navigation soon. The participants in the long-term care facility collided with obstacles such as chairs. This never happened in the VR setting where the courses were cleared, except for the few spawning roadblocks. Finally, they were driving a telerobot with a slight delay on the wireless transmissions of commands, while the VR world responded immediately to gaze inputs. All of these differences point to the importance of not only testing navigation interfaces in VR but also in a real-world setting involving target users.

We have built a platform where turns of the wheels are transferred into steering commands in a game world. This world may both be explored in full 3D wearing a HMD or on a monitor located in front of the wheelchair user. The simulator provides a hardware-in-the-loop realism with regard to the wheelchairs motor and control system and a realistic vibration of the turning wheels [Hansen et al. 2019]. We have used this platform to test a commercial product, "GazeDriver" in front of a large monitor. This product uses a Tobii remote gaze tracker, and the user looks through three small circles above the tracker to steer left, straight, or right. Looking outside the circles makes the wheelchair stop. This simulation set-up allowed the user to be able to attend the full environment via the peripheral vision while driving with gaze. With a large monitor set-up the tests worked well and driving felt much like real gaze-driving. (See Figures 11 and 12.)

\section{CONCLUSION}

This paper presented our ongoing work with the exploration of alternative control of wheelchairs. We conducted an experiment in VR that resulted in significantly better performance and higher user preference for a semi-autonomous waypoint gaze interface compared to a continuous-control and an overlay display. A field study revealed that VR testing had a simplified representation of the steering task, especially with regard to collision avoidance, and also ignored important part of the use context, for instance the feeling of being excluded behind the HMD. Finally, we suggested using a wheelchair platform for a more realistic user experience where hardware-in-the-loop simulations preserves the true characteristics of the wheelchair motion.

\section{ACKNOWLEDGMENTS}

Thanks to the people at Jonstrupvang Long-term Care home who participated in this study. The research was supported by the China Scholarship Council and the Bevica Foundation. Thanks also to Astrid Kofod Trudslev and Nils David Rasamoel for assisting with the study, and to Martin Sølvmose for providing the "GazeDriver".

\section{REFERENCES}

Kohei Arai and Ronny Mardiyanto. 2011. A prototype of electric wheelchair controlled by eye-only for paralyzed user. Fournal of Robotics and Mechatronics 23, 1 (2011), 
Rafael Barea, Luciano Boquete, Luis Miguel Bergasa, Elena López, and Manuel Mazo 2003. Electro-oculographic guidance of a wheelchair using eye movements codification. The International fournal of Robotics Research 22, 7-8 (2003), 641-652.

Christian Bartolein, Achim Wagner, Meike Jipp, and Essameddin Badreddin. 2008 Easing wheelchair control by gaze-based estimation of intended motion. IFAC Proceedings Volumes 41, 2 (2008), 9162-9167.

Laurel J Buxbaum, Mary Ann Palermo, Dina Mastrogiovanni, Mary Schmidt Read Ellen Rosenberg-Pitonyak, Albert A Rizzo, and H Branch Coslett. 2008. Assessment of spatial attention and neglect with a virtual wheelchair navigation task. Journal of Clinical and Experimental Neuropsychology 30, 6 (2008), 650-660.

Mohamad A Eid, Nikolas Giakoumidis, and Abdulmotaleb El Saddik. 2016. A novel eye-gaze-controlled wheelchair system for navigating unknown environments: case study with a person with ALS. IEEE Access 4 (2016), 558-573.

Michael A Elliott, Henrique Malvar, Lindsey L Maassel, Jon Campbell, Harish Kulkarni, Irina Spiridonova, Noelle Sophy, Jay Beavers, Ann Paradiso, Chuck Needham, et al. 2019. Eye-controlled, power wheelchair performs well for ALS patients. Muscle \& nerve 60, 5 (2019), 513-519.

Md. Fahim Bhuyain et Al. 2019. Design and development of an EOG-based system to control electric wheelchairs for people suffering from quadriplegia or quadriparesis. International Conference on Robotics,Electrical and Signal Processing Techniques 0 (2019), 0 .

Mohamed Moncef Ben Khelifa Hachem A. Lamti and Vincent Hugel. 2019. Cerebral and gaze data fusion for wheelchair navigation enhancement: case of distracted users. Robotica 37 (2019), 246-263.

John Paulin Hansen, Astrid Kofod Trudslev, Sara Amdi Harild, Alexandre Alapetite, and Katsumi Minakata. 2019. Providing access to VR through a wheelchair. In Extended Abstracts of the 2019 CHI Conference on Human Factors in Computing Systems. 1-8.

A Harrison, G Derwent, A Enticknap, F Rose, and E Attree. 2000. Application of virtual reality technology to the assessment and training of powered wheelchair users In Proceedings of the 3rd International Conference Disability, Virtual Reality and Associated Technologies.

A Harrison, G Derwent, A Enticknap, FD Rose, and EA Attree. 2002. The role of virtual reality technology in the assessment and training of inexperienced powered wheelchair users. Disability and rehabilitation 24, 11-12 (2002), 599-606.

Robert JK Jacob. 1991. The use of eye movements in human-computer interaction techniques: what you look at is what you get. ACM Transactions on Information Systems (TOIS) 9, 2 (1991), 152-169.

Matthew C Kiernan, Steve Vucic, Benjamin C Cheah, Martin R Turner, Andrew Eisen, Orla Hardiman, James R Burrell, and Margaret C Zoing. 2011. Amyotrophic lateral sclerosis. The lancet 377, 9769 (2011), 942-955.

Sofia Ira Ktena, William Abbott, and A Aldo Faisal. 2015. A virtual reality platform for safe evaluation and training of natural gaze-based wheelchair driving. In 2015 7th International IEEE/EMBS Conference on Neural Engineering (NER). IEEE, 236-239.

Jesse Leaman and Hung Manh La. 2017. A comprehensive review of smart wheelchairs: past, present, and future. IEEE Transactions on Human-Machine Systems 47, 4 (2017), 486-499.

Robert Leeb, Doron Friedman, Gernot R Müller-Putz, Reinhold Scherer, Mel Slater, and Gert Pfurtscheller. 2007. Self-paced (asynchronous) BCI control of a wheelchair in virtual environments: a case study with a tetraplegic. Computational intelligence and neuroscience 2007 (2007)

Chern-Sheng Lin, Chien-Wa Ho, Wen-Chen Chen, Chuang-Chien Chiu, and MauShiun Yeh. 2006. Powered wheelchair controlled by eye-tracking system. Optica Applicata 36 (2006)

Yoshio Matsumotot, Tomoyuki Ino, and Tsukasa Ogsawara. 2001. Development of intelligent wheelchair system with face and gaze based interface. In Proceedings 10th IEEE International Workshop on Robot and Human Interactive Communication. ROMAN 2001 (Cat. No. 01TH8591). IEEE, 262-267.

Daniel Salber and Joëlle Coutaz. 1993. Applying the wizard of oz technique to the study of multimodal systems. In International Conference on Human-Computer Interaction Springer, 219-230.

Kouji Takano Sebastian Halder and Kenji Kansaku. 2018. Comparison of Four Control Methods for a Five-Choice Assistive Technology. Ieej Transactions on Electrical and Electronic Engineering 13 (2018), 1795-1803.

Rosella Spataro, Maria Ciriacono, Cecilia Manno, and Vincenzo La Bella. 2014. The eye-tracking computer device for communication in amyotrophic lateral sclerosis Acta Neurologica Scandinavica 130, 1 (2014), 40-45.

Sophie Stellmach and Raimund Dachselt. 2012. Designing gaze-based user interfaces for steering in virtual environments. In Proceedings of the Symposium on Eye Tracking Research and Applications. ACM, 131-138.

Martin Tall, Alexandre Alapetite, Javier San Agustin, Henrik HT Skovsgaard, John Paulin Hansen, Dan Witzner Hansen, and Emilie Møllenbach. 2009. Gazecontrolled driving. In CHI'09 Extended Abstracts on Human Factors in Computing Systems. ACM, 4387-4392.

Andrew Talone, Thomas Fincannon, David Schuster, Florian Jentsch, and Irwin Hudson. 2013. Comparing physical and virtual simulation use in UGV research: lessons learned from HRI research with two test beds. In Proceedings of the Human Factors and Ergonomics Society Annual Meeting, Vol. 57. SAGE Publications Sage CA: Los Angeles, CA, 2017-2021.

Vildan Tanriverdi and Robert JK Jacob. 2000. Interacting with eye movements in virtual environments. In Proceedings of the SIGCHI conference on Human Factors in Computing Systems. ACM, 265-272.

Chao Wang, Alexey S Matveev, Andrey V Savkin, Tuan Nghia Nguyen, and Hung T Nguyen. 2013. A collision avoidance strategy for safe autonomous navigation of an intelligent electric-powered wheelchair in dynamic uncertain environments with moving obstacles. In 2013 European Control Conference (ECC). IEEE, 4382-4387.

Erik Wästlund, Kay Sponseller, and Ola Pettersson. 2010. What you see is where you go: testing a gaze-driven power wheelchair for individuals with severe multiple disabilities.. In ETRA, Vol. 10. 133-136.

Ginger S Watson, Yiannis E Papelis, and Katheryn C Hicks. 2016. Simulation-based environment for the eye-tracking control of tele-operated mobile robots. In Proceedings of the Modeling and Simulation of Complexity in Intelligent, Adaptive and Autonomous Systems 2016 (MSCIAAS 2016) and Space Simulation for Planetary Space Exploration (SPACE 2016). 1-7.

A. Aldo Faisal William W. Abbott. 2011. Ultra-low cost eyetracking as an highinformation throughput alternative to BMIs. BMC Neuroscience 12 (2011), 103.

Guangtao Zhang and John Paulin Hansen. 2019. Accessible control of telepresence robots based on eye tracking. In Proceedings of the 11th ACM Symposium on Eye Tracking Research \& Applications. 1-3.

Guangtao Zhang and John Paulin Hansen. 2020. People with Motor Disabilities Using Gaze to Control Telerobots. In Extended Abstracts of the 2020 CHI Conference on Human Factors in Computing Systems. 1-9. 